This paper has been retracted. A Retraction notice was published on 1 October 2015 at Energies 2015, 8, 10995; doi:10.3390/en81010995.

Energies 2015, 8, 2769-2787; doi:10.3390/en8042769

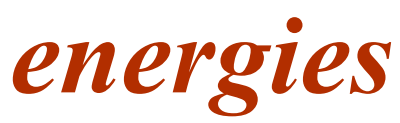

ISSN 1996-1073

www.mdpi.com/journal/energies

Article

\title{
A Robust WLS Power System State Estimation Method Integrating a Wide-Area Measurement System and SCADA Technology
}

Tao Jin ${ }^{1, *}$, Fuliang Chu ${ }^{1}$, Cong Ling ${ }^{2}$ and Daniel Legrand Mon Nzongo ${ }^{1}$

1 College of Electrical Engineering and Automation, Fuzhou University, Fuzhou 350108, China;

E-Mails: fuliangchu@fzu.edu.cn (F.C.); monnzongodaniel@yahoo.fr (M.L.N.)

2 Department of Electrical \& Electronic Engineering, Imperial College London, London SW7 2BT, UK;

E-Mail: cling@imperial.ac.uk

* Author to whom correspondence should be addressed; E-Mail:jintly@fzu.edu.cn;

Tel.: +86-591-2286-6583; Fax: +86-591-2286-6581.

Academic Editor: Brian Agnew

Received: 7 February 2015 /Accepted. 23 March 2015 / Published: 10 April 2015

Abstract: With the development of modern society, the scale of the power system is rapidly increased accordingly, and the framework and mode of running of power systems are trending towards more complexity. It is nowadays much more important for the dispatchers to know exactly the state parameters of the power network through state estimation. This paper proposes a robust power system WLS state estimation method integrating a wide-area measurement system (WAMS) and SCADA technology, incorporating phasor measurements and the results of the traditional state estimator in a post-processing estimator, which greatly reduces the scale of the non-linear estimation problem as well as the number of iterations and the processing time per iteration. This paper firstly analyzes the wide-area state estimation model in detail, then according to the issue that least squares does not account for bad data and outliers, the paper proposes a robust weighted least squares (WLS) method that combines a robust estimation principle with least squares by equivalent weight. The performance assessment is discussed through setting up mathematical models of the distribution network. The effectiveness of the proposed method was proved to be accurate and reliable by simulations and experiments. 
Keywords: wide-area measurement system; state estimation; weighted least squares; bad data detection; distribution network; Huber method

\section{Introduction}

Electric power is essential to modern society. Economic prosperity, national security, and standard of living depend on reliable electric power systems, and it's very important for the power systems to obtain operating condition information about the state of the electric grid [1]. In 1970s, Schweppe first proposed the idea of state estimation in power systems. Power system state estimation constitutes the core of the on-line system monitoring, analysis and control functions [2-5]. State estimation acts like a filter between the raw measurements received from the system and all the application functions that require the most reliable data base for the current system operation state, and it typically includes bad data processing, state estimation solutions, parameter and topology error processing, and other analyses.

In modern power systems, the control center receives the system-wide device information and measurement mainly through a SCADA system [6,7]. Howeyer, the information and measurement data provided by SCADA may not always be accurate and reliable. On the other hand, the collected measurements may not allow direct extraction of the corresponding real-time AC operation state of the system. These concerns drive the development of power system technology. Among all the newly-developed applications that aim at satisfying those new technological demands, the so-called wide area measurement system (WAMS) opens a new avenue for power system stability analysis and control, and it has been attracting increasing attention in recent years, since it is a powerful tool for power system monitoring, protection and control, and has been widely used in the energy management systems of power systems [8-13]. WAMS measurements have high precision and short updating cycles and can measure the node states directly, but due to the high costs of PMU devices and their placement problems, how to utilize WAMS measurements and SCADA information in state estimation effectively must be faced and solved now.

At the same time, the computing processes of power system state estimation generally use the maximum likelihood estimation method, but bad data and outliers exist in the estimation process, whose standardized errors are larger than a pre-established tolerance, and they will affect the accuracy and state estimation effectiveness greatly [14-16], so it is important to research a robust estimation method to prevent the final estimate from being biased. To address this problem, a variety of approaches have been proposed, including linear regression, artificial neural networks, fuzzy pattern matching, Kalman filter techniques, etc. A linearized state estimation algorithm was presented for applications in smart distribution systems $[17,18]$. This method intended to incorporate synchronized phasor measurements into the distribution state estimation by a complex calculation process. As the availability of phasor measurements at substations will increase gradually, the authors of [19,20] studied how the state estimator can be enhanced to handle both the traditional state estimator and the linear state estimator simultaneously. A complex artificial neural network was used to adjust the link weighting in power system bad data analysis and estimation in [21]. Using fuzzy clustering and a pattern matching method, a fuzzy pattern vector for power state estimation was generated based on the 
analog measurement vector in [22]. An adaptive Kalman filter was introduced for real-time power system state estimation in [23], but Kalman filters achieve optimal performance only when the system noise characteristics have known statistical properties. When greater ability and high speed are required in real-time power state estimation, the complexity of these implementations quick increases and it becomes more difficult to complete high-accuracy state estimations of the power system. Consequently, depending on the required ability level, these methods can become impractical for real-time applications.

This paper analyzes in detail the state estimation model by mixed measurements with WAMS and SCADA, and develops a robust WLS state estimation method which can update the weighting factors in each iteration using WAMS data, therefore eliminating bad data and outliers efficiently and thus improving the robustness in the presence of bad measurements. The performance of the proposed state estimation method and the efficiency of the bad data identification were verified by experiments and simulations. The rest of this paper is outlined as follows: in Section 2 we analyze the wide-area state estimation model and the proposed robust WLS theory based on readjusting measurement weights. In Section 3 we focus on performance assessment through simulations and experiments. Conclusions are drawn in Section 4.

\section{A State Estimation Method Integrating WAMS and SCADA}

\subsection{Model Analysis of Power System State Estimation}

In power state estimation methods, the most commonly used measurements are line power flow, bus power injection and voltage magnitude measurements. In some cases, especially for state estimation of distribution buses, the line current magnitude measurements may be taken into consideration too. Where PMUs exist, there will be two new types of measurements utilized by power system state estimation methods. One is voltage phasor measurements which include voltage magnitudes and angles of the system bus, and the other is current phasor measurements which include current magnitudes and angles of the transmission lines or transformers [24]. All types of measurements can be expressed in terms of the system state using Equation (1):

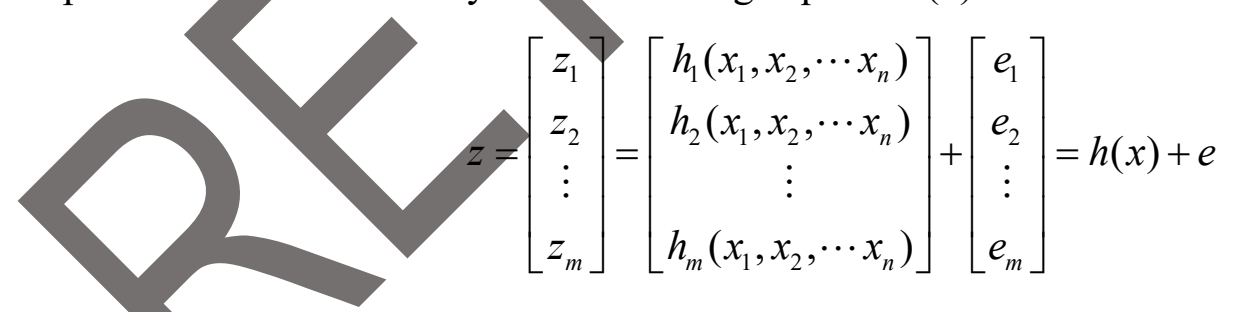

where $z_{i}$ is the measured value, $h_{i}(x)$ is a nonlinear function; $x_{i}$ is the system state vector; including the voltage magnitudes and phase of all the buses excluding the reference bus phase angle; and $e_{i}$ is measurement error. Defining Equation (2) as

$$
J(x)=[z-h(x)]^{T} R^{-1}[z-h(x)]
$$

$R$ is the covariance matrix of the measurement error $e$. The optimization problem of state estimation is to make the aim function $J(x)$ smallest, i.e.:

$$
g(x)=\frac{\partial J(x)}{\partial(x)}=-\frac{\partial h(x)}{\partial(x)} R^{-1}[z-h(x)]=0
$$


here $H(x)=\frac{\partial h(x)}{\partial(x)}$ can be named as Jacobian matrix, and it can be expressed as:

$$
H(x)=\frac{\partial h(x)}{\partial(x)}=\left[\begin{array}{cccc}
\frac{\partial h_{1}(x)}{\partial x_{1}} & \frac{\partial h_{1}(x)}{\partial x_{2}} & \cdots & \frac{\partial h_{1}(x)}{\partial x_{n}} \\
\frac{\partial h_{2}(x)}{\partial x_{1}} & \frac{\partial h_{2}(x)}{\partial x_{2}} & \cdots & \frac{\partial h_{2}(x)}{\partial x_{n}} \\
\vdots & \vdots & \ddots & \\
\frac{\partial h_{m}(x)}{\partial x_{1}} & \frac{\partial h_{m}(x)}{\partial x_{n}} & \cdots & \frac{\partial h_{m}(x)}{\partial x_{n}}
\end{array}\right]
$$

where $i$ is the iteration index; $x_{i}$ is the solution vector at the ith iteration. Using mathematical transformations such as Taylor series expansion and the Newton iterative method, we can obtain Equation (5) from the above equations:

$$
H^{T}\left(x_{i}\right) R^{-1}\left[z-h\left(x_{i}\right)\right]=H^{T}\left(x_{i}\right) R^{-1} H\left(x_{i}\right)\left(x_{i+1}-x_{i}\right)
$$

Defining $\Delta x_{i}=x_{i+1}-x_{i}$, at the ith iteration, it is decomposed into its triangular factors, and the following equation should be solved using related theory:

$$
\left[G\left(x_{i}\right)\right] \Delta x_{i}=H^{T}\left(x_{i}\right) R^{-1}\left[z-h\left(x_{i}\right)\right]
$$

$G\left(x_{i}\right)=\frac{\partial g\left(x_{i}\right)}{\partial(x)}=H^{T}\left(x_{i}\right) R^{-1} H\left(x_{i}\right)$ is called the gain matrix. It is sparse positive definite and symmetric if the system is fully observable. State estimation inyolves the iterative solution of the equations given by Equation (5), and we need to get initial state estimation data first so the related equivalent circuit of different power system components, admittance matrix, power injection, power flow, and so on should be researched first.

In a power system, transmission lines can be seen as middle-length transmission and the conductance need not be considered, then the equivalent circuit of transmission lines is as shown in Figure 1. In this figure, $Z$ indicates impedance and $\frac{Y}{2}$ indicates admittance. Based on Kirchhoff's law,

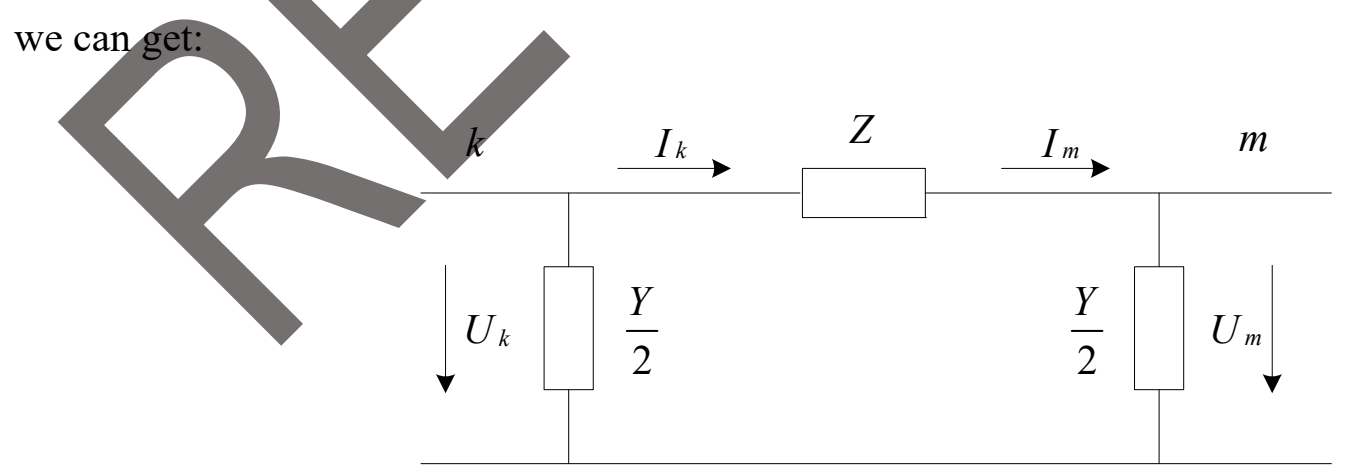

Figure 1. Equivalent circuit for transmission line. 


$$
\left[\begin{array}{c}
U_{k} \\
I_{k}
\end{array}\right]=\left[\begin{array}{cc}
1+\frac{Z Y}{2} & Z \\
Y\left(1+\frac{Z Y}{4}\right) & 1+\frac{Z Y}{2}
\end{array}\right]\left[\begin{array}{c}
U_{m} \\
I_{m}
\end{array}\right]
$$

According to tap changing and phase shifting transformers, Figure 2 is the typical equivalent circuit. The two transformer terminal buses are commonly designated as the impedance side and the tap side bus respectively.

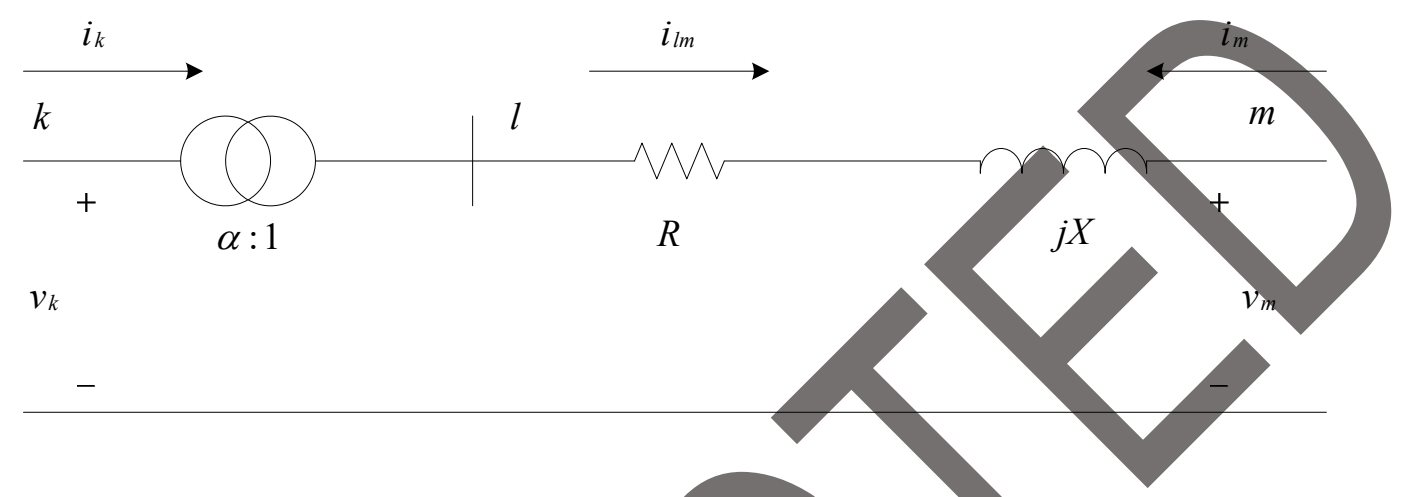

Figure 2. Equivalent circuit for a transformer.

From a transformer's features, it is easy to know $i_{l_{m}}=a \cdot i_{k}, v_{k}=v_{k} / a$. Defining the admittance of the branch $l-m$ is $y$, then we can get:

$$
\left[\begin{array}{c}
i_{l m} \\
i_{m}
\end{array}\right]=\left[\begin{array}{cc}
y & -y \\
-y & y
\end{array}\right]\left[\begin{array}{c}
v_{1} \\
v_{m}
\end{array}\right]
$$

If the tap ratio $a$ is complex, through substituting calculations, the above equation can be turned into:

$$
\left[\begin{array}{c}
i_{k} \\
i_{m}
\end{array}\right]=\left[\begin{array}{cc}
y /|a|^{2} & -y / a^{*} \\
-y / a & y
\end{array}\right]\left[\begin{array}{l}
v_{k} \\
v_{m}
\end{array}\right]
$$

Generators are normally seen in the power system. The equivalent circuit of generators is divided into voltage and current equivalent circuits as shown in Figure $3 \mathrm{a}$ and Figure $3 \mathrm{~b}$.

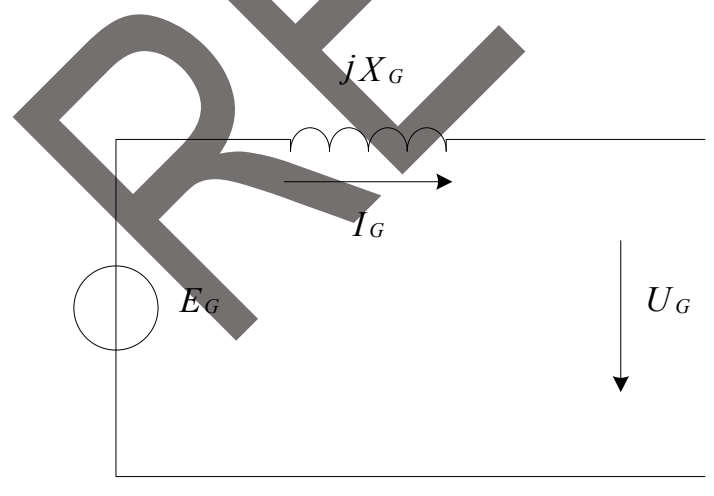

(a)

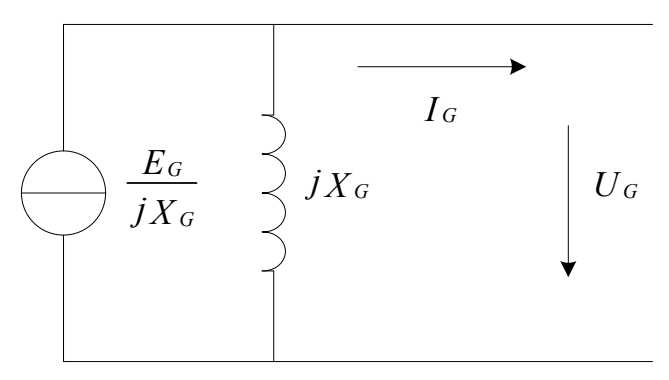

(b)

Figure 3. Equivalent circuit for a generator. (a) Voltage equivalent circuit for a generator; (b) Current equivalent circuit for a generator. 
The reactance of generators is expressed by Equation (10), where $X_{G} \%$ is the reactance percent value of generators; $U_{G N}$ is the rated voltage of generators; $S_{N}$ is the nominal power of generators:

$$
X_{G}=\frac{X_{G} \% U_{G N}^{2}}{100 S_{N}}
$$

Using Kirchhoff's current law at each bus to build the network model for the entire power system, the following equation can be obtained:

$$
I=\left[\begin{array}{c}
i_{1} \\
i_{2} \\
\vdots \\
i_{N}
\end{array}\right]=\left[\begin{array}{cccc}
Y_{11} & Y_{12} & \cdots & Y_{1 N} \\
Y_{21} & Y_{22} & \cdots & Y_{2 N} \\
\vdots & \vdots & \ddots & \vdots \\
Y_{N 1} & Y_{N 2} & \cdots & Y_{N N}
\end{array}\right]\left[\begin{array}{c}
v_{1} \\
v_{2} \\
\vdots \\
v_{N}
\end{array}\right]=Y \bullet V
$$

where $i_{k}, v_{k}$ are respectively the net current injection phasor, the voltage phasor at bus $k$. Matrix $Y$ is the bus admittance matrix, and when considering the transformer, the following equation can be given:

$$
\begin{aligned}
& Y_{k k}^{\text {new }}=Y_{k k}+y_{t} /|a|^{2} \\
& Y_{k m}^{\text {new }}=Y_{k m}-y_{t} / a \\
& Y_{m k}^{\text {new }}=Y_{m k}-y_{t} / a \\
& Y_{m m}^{\text {new }}=Y_{m m}+y_{t}
\end{aligned}
$$

Assuming Figure 4 is the general two-port model for the power network branch. In the figure $g_{i j}+j b_{i j}$ is the admittance of the series branch connecting buses $i$ and $j, g_{s i}+j b_{s i}$ is the admittance of the shunt branch connected at bus $i$, then we can get all kinds of measurements $h(x)$ which are expressed by voltage, angle, and other network parameters.

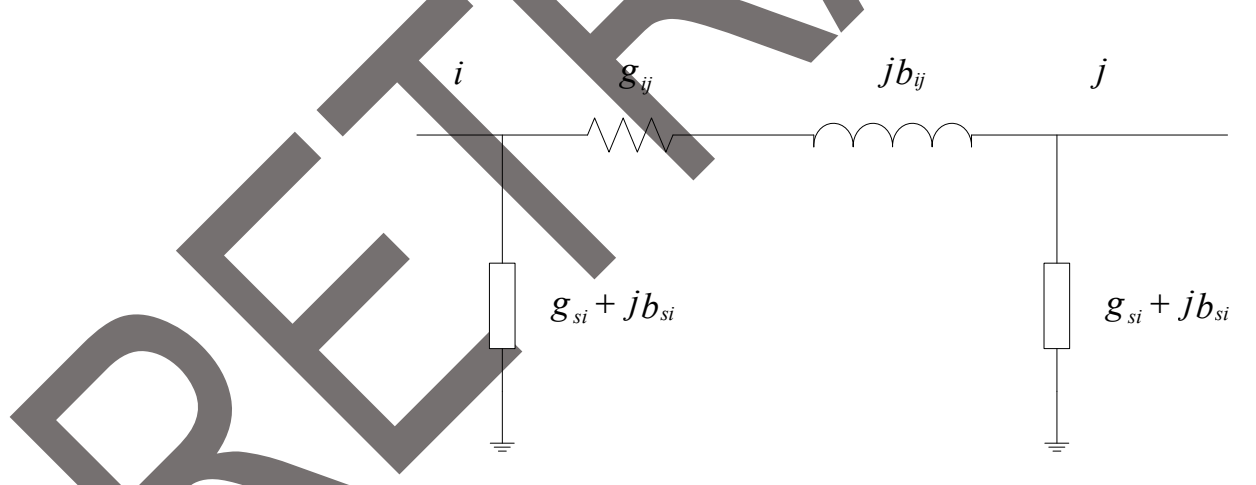

Figure 4. Two-port model of power network branch.

In Figure 4, the $v_{i}, v_{j}$ can be shown as Equation (13) with voltage amplitude $V_{i}, V_{j}$ and the phase angle $\theta_{i}, \theta_{j}$ :

$$
\begin{aligned}
& v_{i}=V_{i}\left(\cos \theta_{i}+j \sin \theta_{i}\right) \\
& v_{j}=V_{j}\left(\cos \theta_{j}+j \sin \theta_{j}\right)
\end{aligned}
$$

Based on Kirchhoff's current law, that is $i_{i}=\sum_{i=1}^{N} Y_{i j} v_{j}$, the power can be calculated with the following equation: 


$$
P_{i}+j Q_{i}=v_{i} \cdot i_{i}^{*}
$$

From the above equations, we get that the real power injection and reactive power injection at bus $i$ are:

$$
\begin{aligned}
& P_{i}=V_{i} \sum_{j \in N_{i}} V_{j}\left(G_{i j} \cos \theta_{i j}+B_{i j} \sin \theta_{i j}\right) \\
& Q_{i}=V_{i} \sum_{j \in N_{i}} V_{j}\left(G_{i j} \sin \theta_{i j}-B_{i j} \cos \theta_{i j}\right)
\end{aligned}
$$

where $\theta_{i j}=\theta_{i}-\theta_{j}, G_{i j}, B_{i j}$ is in matrix form. From Figure 4, we also can get:

$$
\begin{aligned}
& i_{i j}=\left(v_{i}^{*}-v_{j}^{*}\right) y_{i j}+j v_{i}^{*}\left(g_{s i}+j b_{s i}\right) \\
& P_{i j}+j Q_{i j}=v_{i}^{*} i_{i j}^{*}
\end{aligned}
$$

so, the real power flow, reactive power flow and line current flow from bus $i$ to $j$ can be described as follows:

$$
\begin{aligned}
& P_{i j}=V_{i}^{2}\left(g_{s i}+g_{i j}\right)-V_{i} V_{j}\left(g_{i j} \cos \theta_{i j}+b_{i j} \sin \theta_{i j}\right) \\
& Q_{i j}=-V_{i}^{2}\left(b_{s i}+b_{i j}\right)-V_{i} V_{j}\left(g_{i j} \sin \theta_{i j}-b_{i j} \cos \theta_{i j}\right) \\
& I_{i j}=\frac{\sqrt{P_{i j}^{2}+Q_{i j}^{2}}}{V_{i}}
\end{aligned}
$$

Then we can calculate the partial differential elements corresponding to active power injection measurements, reactive power injection measurements, active power flow measurements, and so on. The following equation is an example of partial differential results corresponding to active power injection measurements:

$$
\begin{aligned}
& \frac{\partial P_{i}}{\partial \theta_{i}}=\sum_{j=1}^{N} V_{i} V_{j}\left(-G_{i j} \sin \theta_{i j}+B_{i j} \cos \theta_{i j}\right)-V_{i}^{2} B_{s i} \\
& \frac{\partial P_{i}}{\partial \theta_{j}}=V_{i} V_{j}\left(-G_{i j} \sin \theta_{i j}+B_{i j} \cos \theta_{i j}\right) \\
& \frac{\partial P_{i}}{\partial V_{j}}=\sum_{j=1}^{N} V_{j}\left(G_{i j} \cos \theta_{i j}+B_{i j} \sin \theta_{i j}\right)+V_{i} G_{s i} \\
& \frac{\partial P_{i}}{\partial V_{j}}=V_{i}\left(G_{i j} \cos \theta_{i j}+B_{i j} \sin \theta_{i j}\right)
\end{aligned}
$$

Through the above analysis and calculations, we can get $H(x), G\left(x_{i}\right)$ and other parameters, then an iterative solution state estimation of power network will be completed.

\subsection{Proposed Power System State Estimation Method}

PMUs are used to measure data such as voltages, current, angle and frequency, and can provide real-time information about the state of large portions of national and transnational grids and allow obtaining a state estimate which is a true snapshot of the power system, making the technology for real-time supervision of power systems clearly at hand [25], but because of the high relative cost and 
inconvenience of installation, we can not install PMUs in each part of power grid, and we still need state estimation technology using SCADA and other technologies to get the full state of power grid, and the algorithm and application of state estimation will still need to be explored, proposed and tested in the coming years. This section will analyze a power network state estimation method which includes PMUs.

As shown in Figure 5, when there is a PMU in $i$ node and no PMU in node $j$, the following relationship exists from circuit theory:
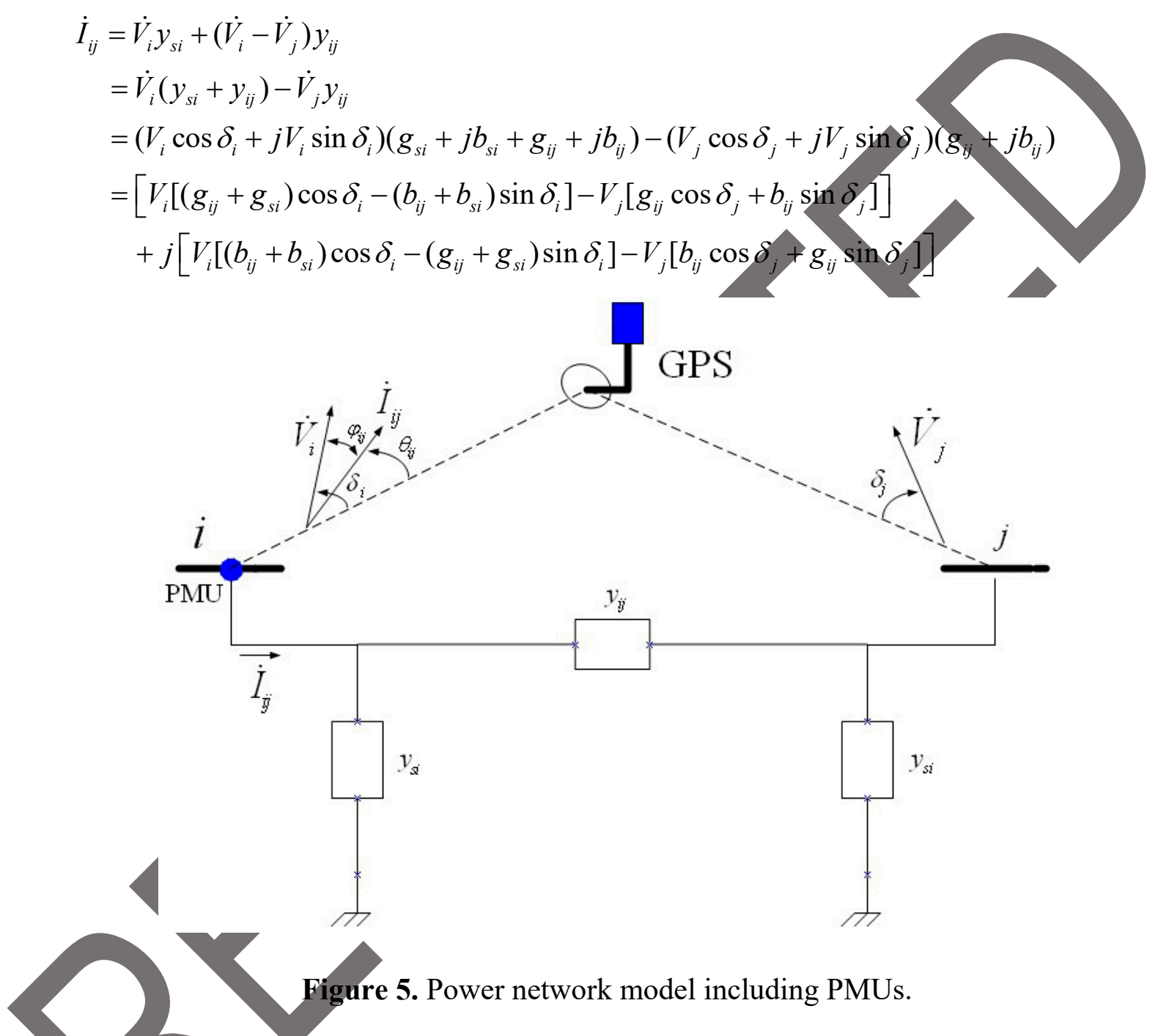

where $y_{i j}=g_{i j}+j b_{i j}$ is the series admittance of branch $i j, y_{s i}=g_{s i}+j b_{s i}$ is the shunt admittance relative to ground of node $i, \dot{V}_{i}=V_{i} \angle \delta_{i}$ and $\dot{V}_{j}=V_{j} \angle \delta_{j}$ are respectively the voltage phasors of node $i$ and node $j$.

Since there is a PMU in $i$, the node voltage amplitude $V_{i}$ and phase $\delta_{i}$ of node $i$, current amplitude $I_{i j}$ and phase $\theta_{i j}$ of branch $i j$ can be directly obtained, so if we define $A_{i j}, B_{i j}, \mathrm{C}_{i j}$ as follows:

$$
\begin{aligned}
& A_{i j}=\left(g_{i j}+g_{s i}\right)^{2}+\left(b_{i j}+b_{s i}\right)^{2} \\
& B_{i j}=g_{i j}^{2}+b_{i j}^{2} \\
& C_{i j}=\left(b_{s i} g_{i j}-g_{s i} b_{i j}\right) \sin \left(\delta_{i}-\delta_{j}\right)-\left(g_{i j}^{2}+b_{i j}^{2}+b_{s i} b_{i j}+g_{s i} g_{i j}\right) \cos \left(\delta_{i}-\delta_{j}\right)
\end{aligned}
$$

Then we can get Equation (21) from Equation (19): 


$$
\begin{aligned}
& I_{i j}=\sqrt{A_{i j} V_{i}^{2}+B_{i j} V_{j}^{2}+2 C_{i j} V_{i} V_{j}} \\
& \theta_{i j}=\arctan \left(\frac{V_{i}\left[\left(b_{i j}+b_{s i}\right) \cos \delta_{i}-\left(g_{i j}+g_{s i}\right) \sin \delta_{i}\right]-V_{j}\left[b_{i j} \cos \delta_{j}+g_{i j} \sin \delta_{j}\right]}{V_{i}\left[\left(b_{i j}+b_{s i}\right) \cos \delta_{i}-\left(g_{i j}+g_{s i}\right) \sin \delta_{i}\right]-V_{j}\left[b_{i j} \cos \delta_{j}+g_{i j} \sin \delta_{j}\right]}\right)
\end{aligned}
$$

To have the state estimation of a power network, one of the key problems is how to solve the Jacobian matrix. From Figure 5, the transposed matrix of the Jacobian matrix can be described by:

$$
H^{T}=\left[\begin{array}{llllllll}
\frac{\partial P_{i j}}{\partial \delta} & \frac{\partial Q_{i j}}{\partial \delta} & \frac{\partial P_{i}}{\partial \delta} & \frac{\partial Q_{i}}{\partial \delta} & \frac{\partial \delta_{i}}{\partial \delta} & \frac{\partial V_{i}}{\partial \delta} & \frac{\partial I_{i j}}{\partial \delta} & \frac{\partial \theta_{i j}}{\partial \delta} \\
\frac{\partial P_{i j}}{\partial V} & \frac{\partial Q_{i j}}{\partial V} & \frac{\partial P_{i}}{\partial V} & \frac{\partial Q_{i}}{\partial V} & \frac{\partial \delta_{i}}{\partial V} & \frac{\partial V_{i}}{\partial V} & \frac{\partial I_{i j}}{\partial V} & \frac{\partial \theta_{i j}}{\partial V}
\end{array}\right]
$$

From Equation (21), we can see the Jacobian matrix is composed of partial derivatives, and all these partial derivatives would be calculated based on Kirchhoff's laws and circuit theory. For example, some branch current partial derivatives and branch phase partial derivatives can be calculated as:

$$
\begin{gathered}
\frac{\partial I_{i j}}{\partial \delta_{i}}=\frac{D_{i j} V_{i} V_{j}}{\sqrt{E_{i j}}} ; \frac{\partial I_{i j}}{\partial \delta_{j}}=\frac{D_{i j} V_{i} V_{j}}{\sqrt{E_{i j}}}=-\frac{\partial I_{i j}}{\partial \delta_{i}} \\
\frac{\partial I_{i j}}{\partial V_{i}}=\frac{A_{i j} V_{i}+C_{i j} V_{j}}{\sqrt{E_{i j}}} ; \frac{\partial I_{i j}}{\partial V_{j}}=\frac{B_{i j} V_{j}+C_{i j} V_{i}}{\sqrt{E_{i j}}} \\
\frac{\partial \theta_{i j}}{\partial \delta_{i}}=\frac{A_{i j} V_{i}^{2}+C_{i j} V_{i} V_{j}}{\frac{E_{i j}}{\partial \delta_{j}}=\frac{B_{i j} V_{j}^{2}+C_{i j} V_{i} V_{j}}{E_{i j}}} \\
\frac{\partial \theta_{i j}}{\partial V_{i}}=\frac{D_{i j} V_{j}}{E_{i j}} ; \frac{\partial \theta_{i j}}{\partial V_{j}}=-\frac{D_{i j} V_{i}}{E_{i j}}
\end{gathered}
$$

where $D_{i j}=\left(g_{i j}+g_{s i}\right) \beta_{i j}+\left(b_{i j}+b_{s i}\right) \alpha_{i j}, E_{i j}=A_{i j} V_{i}^{2}+B_{i j} V_{j}^{2}+2 C_{i j} V_{i} V_{j}$. As concerned as above, the key issue is to calculate Equation (2), defining the measurement residual error $v=z_{i}-h_{i}(\hat{x})$. In each iteration, there is the equation:

$$
\begin{aligned}
& \left.J(x)=[\Delta z-H \Delta x)^{T}\right] R^{-1}[\Delta z-H \Delta x]=\left|\left[R^{-\frac{1}{2}} \Delta z-R^{-\frac{1}{2}} H \Delta x\right]\right|^{2} \\
& \text { Defining } \Delta \hat{z}=R^{-\frac{1}{2}} \Delta z, \hat{H}=R^{-\frac{1}{2}} H, Q^{T} Q=I, I \text { is unit matrix, and } Q \hat{H}=\left[\begin{array}{c}
U \\
0
\end{array}\right] . U \text { is a upper }
\end{aligned}
$$
triangular matrix, so:

$$
J(x)=\left|\left[R^{-\frac{1}{2}} \Delta z-R^{-\frac{1}{2}} H \Delta x\right]\right|^{2}=\left\|\Delta y_{1}-U \Delta x\right\|^{2}+\left\|\Delta y_{2}\right\|^{2}
$$

where $Q \Delta \hat{z}=\left[\begin{array}{l}\Delta y_{1} \\ \Delta y_{2}\end{array}\right]$. When $\Delta y_{1}-U \Delta x=0$, i.e., $\Delta y_{1}=U \Delta x$, we get the smallest $J(x)$, then we get the optimization result, so the criterion function of the proposed algorithm is $\sum_{i=1}^{n} P_{i} v_{i}^{2}=\min , P_{i}$ is the weight of $z_{i}$, and the normal equation used to solve $\hat{x}$ can be written as: 


$$
H^{T} P H \hat{x}-H^{T} P z=0
$$

Through the Huber robust method, there is $\psi\left(v_{i}\right)=\rho\left(v_{i}\right), \sum_{i=1}^{n} w_{i} v_{i} h_{i}=0, w_{i}$ is weight factor. Defining:

$$
\begin{aligned}
& \psi(u)=\left\{\begin{array}{cc}
-k & u<-k \\
u & -k \leq u \leq k \\
k & u>k
\end{array}\right. \\
& w(u)=\left\{\begin{array}{cc}
1 & |u| \leq k \\
k \bullet \operatorname{sign}(u) & |u|>k
\end{array}\right.
\end{aligned}
$$

where $u=\left(l-h_{i}{ }^{T} \hat{x}\right) / s$. When $|u| \leq k, s=\hat{\sigma} ;|u|>k, s=\operatorname{med}_{i} d\left|l_{i}-h_{i}{ }^{2} \hat{x}\right|$. By mathematical transform, the equivalent weight can be obtained as $\bar{P}=\left\{P_{i} w_{i}\right\} . \bar{P}$ is a readjusting weigh matrix, so $\hat{x}$

$$
\hat{x}=\left(H^{T} \bar{P} H\right)^{-1} H^{T} \bar{P} z
$$

Then a robust state estimation method which can resist bad data and outliers efficiently is obtained by the technique of adjusting the equivalent weight.

\section{Performance Evaluation}

\subsection{Computer Simulation}

The proposed robust state estimate method was tested using simulated measurements on different size systems such as IEEE 14, 30, 57 and 118 bus systems. Figure 6 is the measurement configuration diagram of the IEEE 14 bus system. From this figure, the simulation bus system has in total 14 buses and 20 branches, which has PMUs in bus 2, bus 6, bus 7, bus 9, and bus 13. With PMU measurement, we can directly get the node voltage magnitudes, node voltage phase angles, and branch currents. In order to simulate Gaussian distribution error of the measurement value:

$$
z_{i}^{\text {mea }}=z_{i}^{\text {true }}+\operatorname{rand} \times \sigma_{i}
$$

where $z_{l}^{\text {mea }}$ is measurement value, $z_{i}^{\text {true }}$ is the true value calculated by load flow software, rand is a function which can generate a Gaussian distribution random number, $\sigma_{i}$ is the standard deviation (STD). As we know, the confidence interval $(\mu-3 \sigma, \mu-3 \sigma)$ includes the $99.7 \%$ confidence rate in the Gaussian distribution condition, then:

$$
\sigma_{i}=\frac{\mu_{i} \times \text { error } \%}{3 \times 100}=\frac{z_{i}^{\text {true }} \times \text { error } \%}{3 \times 100}
$$

If bad data and outliers exist in the system, defining $z_{i}^{\text {bad }}$ as bad measurement data, error\% is measurement error rate, then the gross error can be written as:

$$
z_{i}^{\text {bad }}=z_{i}^{\text {true }}+b_{i} \sigma_{i}
$$

where $b_{i}$ is the coefficient of $\sigma_{i}$, and in our simulations $b_{i}>3$. 


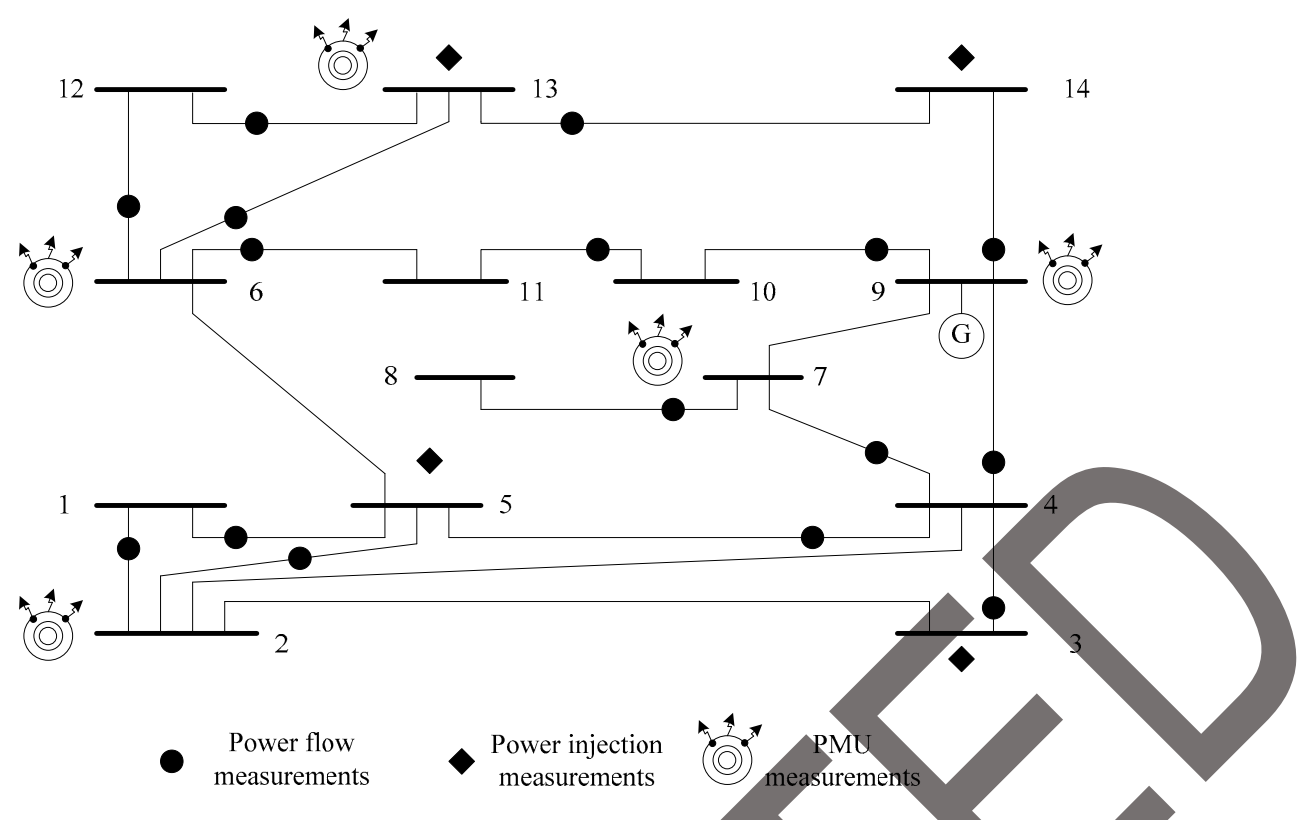

Figure 6. Simulation measurement configuration of the IEEE 14 bus system.

As Table 1 shows, four case studies based on Figure 6 are analyzed to check the estimation accuracy and computational efficiency of the proposed state estimation method. In Table 1, the first column indicates the case number, and the second column shows the measurement configurations of each simulation case. The third and the fourth columns indicate the number of measurements and state vectors. Column 5 shows the redundancy which can be calculated by column 3 and column 4 .

Table 1. Parameter configuration of the cases used in simulations.

\begin{tabular}{|c|c|c|c|c|}
\hline Case No. & ration & $\begin{array}{c}\text { Number of } \\
\text { measurements }\end{array}$ & $\begin{array}{c}\text { Number of } \\
\text { state }\end{array}$ & Redundancy \\
\hline 1 & ditional measurements & 44 & 28 & 1.57 \\
\hline 2 & $S$ and $P M U$ & 50 & 28 & 1.78 \\
\hline & $\begin{array}{l}\text { ments and PMUs } \\
6,7,9\end{array}$ & 50 & 28 & 1.78 \\
\hline & $\begin{array}{l}\text { onal measurements and PMUs } \\
\text { in bus } 2,6,7,9,13\end{array}$ & 50 & 28 & 1.78 \\
\hline
\end{tabular}

The following four cases will be discussed to illustrate the proposed estimation method's performance. Statistic analysis results of relative errors between the state estimation solutions will be presented for comparison of the effects of the different cases.

(1) Case 1: just includes traditional measurements. Power flows in 1-2, 1-5, 2-5, 3-4, 4-5, 4-7, 4-9, 6-11, 6-12, 6-13, 7-8, 7-9, 9-10, 9-14, 10-11, 12-13 and 13-14. Power injections in buses 3, 5, 13 and 14 . Voltage amplitude in bus 1.

(2) Case 2: Power flows in 4-5, 4-7, 4-9, 7-9, 10-11, 12-13 and 13-14. Power injections in buses $3,5,13$ and 14. PMU measurements in bus 2, 7, and 9 . 
(3) Case 3: Power flows in 4-9, 10-11 and 12-13. Power injections in buses 3, 5, and 14. PMU measurements in bus 2, 6, 7, and 9 .

(4) Case 4: Power flows in 10-11. Power injections in buses 5. PMU measurements in bus 2, 6, 7, 9, and 13 .

To easily calculate and intuitively display the results, usually it is better to transform the active, reactive power injection measurements $P_{i}, Q_{i}$ and active, reactive power flow $P_{i j}, Q_{i j}$ to per-unit values. In this paper, we selected the reference value as $100 \mathrm{MW}$ or $100 \mathrm{MVAr}$ to transform the above data to per-unit system, and phase angle's units are degrees $\left({ }^{\circ}\right)$. All other measurements' units are p.u. Assuming the traditional measurements error ranges from $1 \%$ to 3\%. Measurements from PMUs have a high accuracy, and their amplitude errors are from $0.1 \%$ to $1 \%$, and the range of phase angle is from $0.01^{\circ}$ to $0.05^{\circ}$. Tolerance to converge is set to $10^{-4}$, meaning that if there is:

$$
\max \left|\Delta x_{k}\right| \leq \varepsilon=0.0001
$$

the simulation program calculation will finish its iteration and we get the state estimation results. Since it is not possible to show the results of the proposed state estimation method for all the system states, two calculation examples will be presented in the following. Similar results are obtained for all state variables in all the simulations. Table 2 is the bus voltage estimation results of the cases used in simulations. The second column indicates the true number of the bus voltage amplitude, and the third, fourth, fifth and the sixth columns show voltage state estimate values of different cases. Table 3 is the bus voltage estimation results of the cases used in the simulations.

Table 2. Bus voltage estimation of the cases used in simulations.

\begin{tabular}{cccccc}
\hline \multirow{2}{*}{ Bus Voltage } & True value & \multicolumn{3}{c}{ Bus voltage amplitude estimation of simulation cases } \\
\cline { 2 - 5 } & Case 1 & Case 2 & Case 3 & Case 4 \\
\hline$V_{1}$ (p.u.) & 1.0600 & 1.05663 & 1.05755 & 1.0622 & 1.0605 \\
$V_{2}$ (p.u.) & 1.0450 & 1.0417 & 1.0425 & 1.0472 & 1.0455 \\
$V_{3}$ (p.u.) & 0.9996 & 0.9950 & 0.9967 & 1.0017 & 0.9997 \\
$V_{4}$ (p.u.) & 1.0016 & 0.9982 & 0.9992 & 1.0036 & 1.0020 \\
$V_{5}$ (p.u.) & 1.0081 & 1.0056 & 1.0057 & 1.0102 & 1.0085 \\
$V_{6}$ (p.u.) & 0.9805 & 0.9781 & 0.9784 & 0.9824 & 0.9809 \\
$V_{7}$ (p.u.) & 0.9907 & 0.9874 & 0.9885 & 0.9927 & 0.9912 \\
$V_{8}$ (p.u.) & 1.0207 & 1.0175 & 1.0184 & 1.0226 & 1.0211 \\
$V_{9}$ (p.u.) & 0.9678 & 0.9654 & 0.9655 & 0.9697 & 0.9683 \\
$V_{10}$ (p.u.) & 0.9619 & 0.9571 & 0.9597 & 0.9639 & 0.9624 \\
$V_{11}$ (p.u.) & 0.9674 & 0.9640 & 0.9651 & 0.9693 & 0.9678 \\
$V_{12}$ (p.u.) & 0.9644 & 0.9620 & 0.9624 & 0.9663 & 0.9648 \\
$V_{13}$ (p.u.) & 0.9594 & 0.9571 & 0.9574 & 0.9613 & 0.9598 \\
$V_{14}$ (p.u.) & 0.9444 & 0.9421 & 0.9423 & 0.9463 & 0.9450 \\
\hline
\end{tabular}


Table 3. Bus voltage angle estimation of the cases used in simulations.

\begin{tabular}{cccccc}
\hline \multirow{2}{*}{$\begin{array}{c}\text { Angle of bus } \\
\text { voltage }\end{array}$} & True value & \multicolumn{3}{c}{ Bus voltage angle estimation of simulation cases } \\
\cline { 2 - 6 } & & Case 1 & Case 2 & Case 3 & Case 4 \\
\hline$\delta_{1}\left(^{\circ}\right)$ & 0.0000 & 0.0276 & 0.0077 & 0.0073 & 0.0252 \\
$\delta_{2}\left(^{\circ}\right)$ & -5.0125 & -5.0103 & -4.9779 & -4.9869 & -4.9886 \\
$\delta_{3}\left(^{\circ}\right)$ & -12.7398 & -12.8163 & -12.6647 & -12.7642 & -12.7926 \\
$\delta_{4}\left(^{\circ}\right)$ & -10.1673 & -10.1864 & -10.1399 & -10.1563 & -10.1612 \\
$\delta_{5}\left(^{\circ}\right)$ & -8.6505 & -8.6761 & -8.6238 & -8.6375 & -8.6397 \\
$\delta_{6}\left(^{\circ}\right)$ & -14.9247 & -14.9321 & -14.9019 & -14.9165 & -14.9167 \\
$\delta_{7}\left(^{\circ}\right)$ & -13.6736 & -13.6712 & -13.6519 & -13.6623 & -13.6743 \\
$\delta_{8}\left(^{\circ}\right)$ & -13.6636 & -13.6713 & -13.6630 & -13.6731 & -13.6847 \\
$\delta_{9}\left(^{\circ}\right)$ & -15.5817 & -15.5364 & -15.5535 & -15.5655 & -15.5862 \\
$\delta_{10}\left({ }^{\circ}\right)$ & -15.8072 & -15.7407 & -15.7757 & -15.7887 & -15.8105 \\
$\delta_{11}\left({ }^{\circ}\right)$ & -15.5225 & -15.4342 & -15.4944 & -15.5071 & -15.5275 \\
$\delta_{12}\left(^{\circ}\right)$ & -15.9298 & -15.8405 & -15.9049 & -15.9222 & -15.9195 \\
$\delta_{13}\left(^{\circ}\right)$ & -16.0145 & -15.9170 & -15.9912 & -16.0055 & -16.0033 \\
$\delta_{14}\left({ }^{\circ}\right)$ & -16.9687 & -16.8838 & -16.9349 & -16.9401 & -16.9634 \\
\hline
\end{tabular}

Figure 7 and Figure 8 are the bus voltage amplitude and angle estimate errors using the data from Table 1 and Table 2. From the figure, we can see that case 1 has the worst state estimation effect and case 4 has the best estimation effect. This shows that when adding PMU data to conventional SCADA measurement inputs, the state estimator will improve its convergence speed and accuracy.
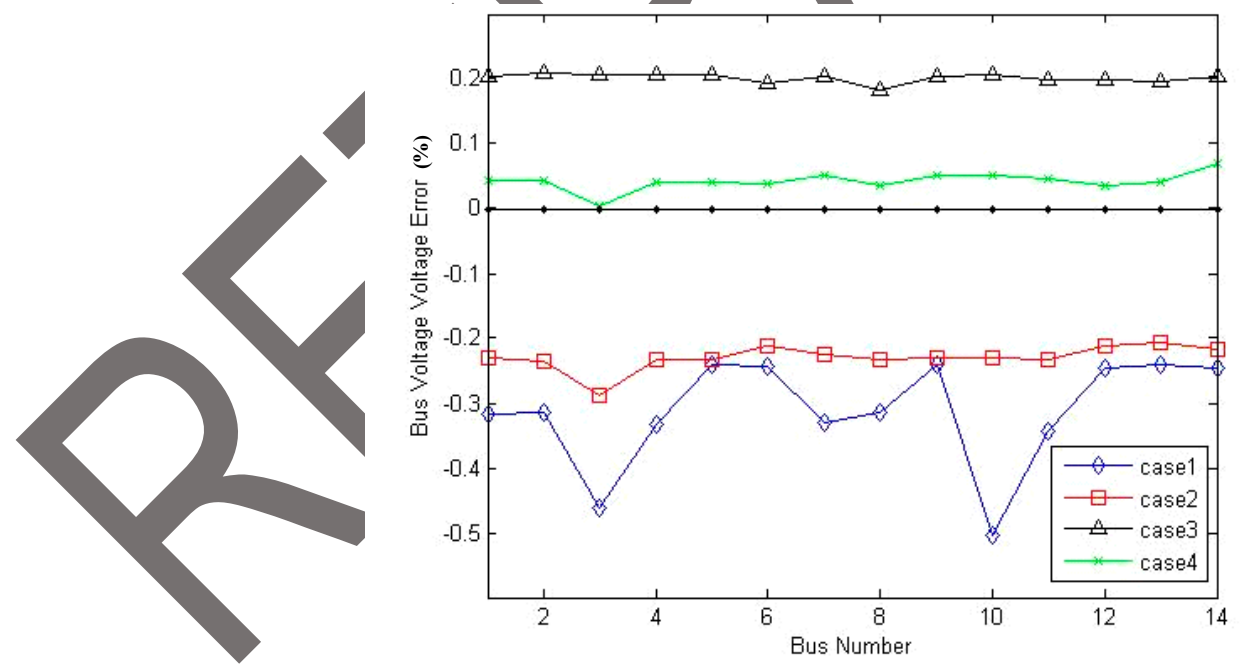

Figure 7. Bus voltage amplitude estimate error of simulation cases. 


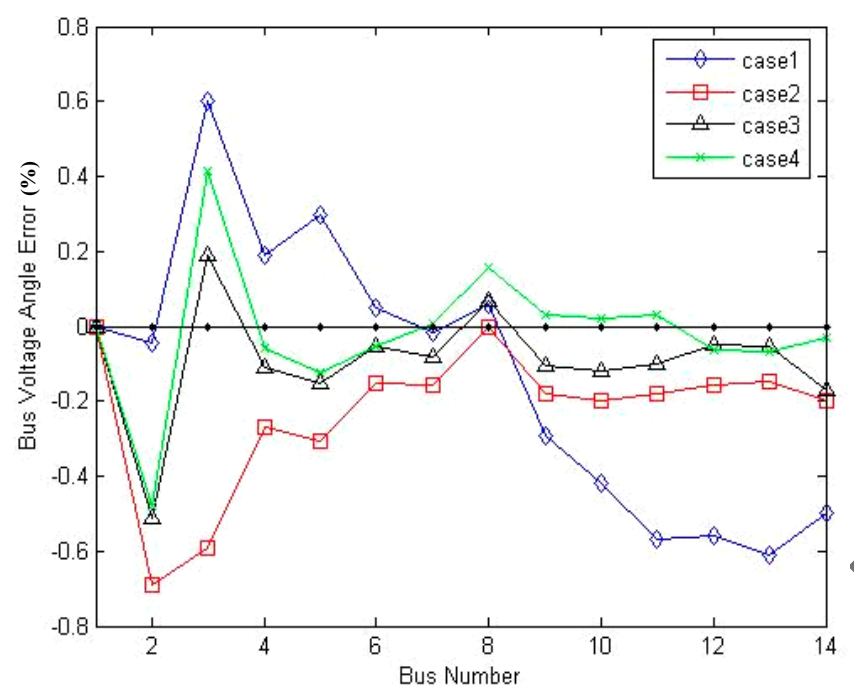

Figure 8. Bus voltage angle estimate error of simulation cases.

The proposed robust state estimation method also shows strong ability to resist bad data and outliers. In the simulations when gross errors are input and the measurement vector contains bad data, using ANOVA analysis, the proposed algorithm can identify the bad data accurately through iterations. Table 4 is the bad data identification results of the cases used in the simulations.

Table 4. Bad data identification results of the cases used in simulations.

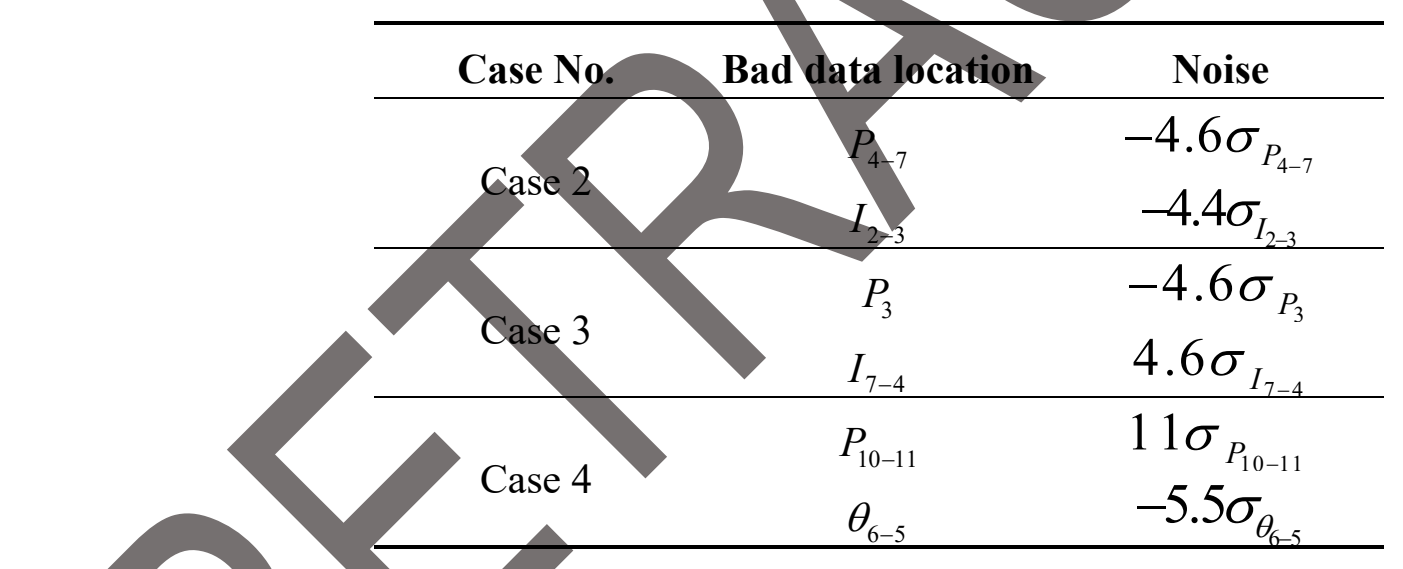

3.2. Hardware Application Experiments

The proposed robust WLS method was implemented on a four-node experiment test system as shown in Figure 9. PSCAD is used to simulate the power system parameters such as active power, reactive power, etc. The simulated results of PSCAD are imported to a RTDS system. SCADA measurements are arranged in bus 1 and bus 3. As we know, due to the lack of a reliable real-time scale between test results of different locations, SCADA monitoring data is valid only in the local system, and it is difficult to detect and analyze the dynamic state of an entire power system just with SCADA, so we also used one PMU in the experiment, which is set to bus 1 . 


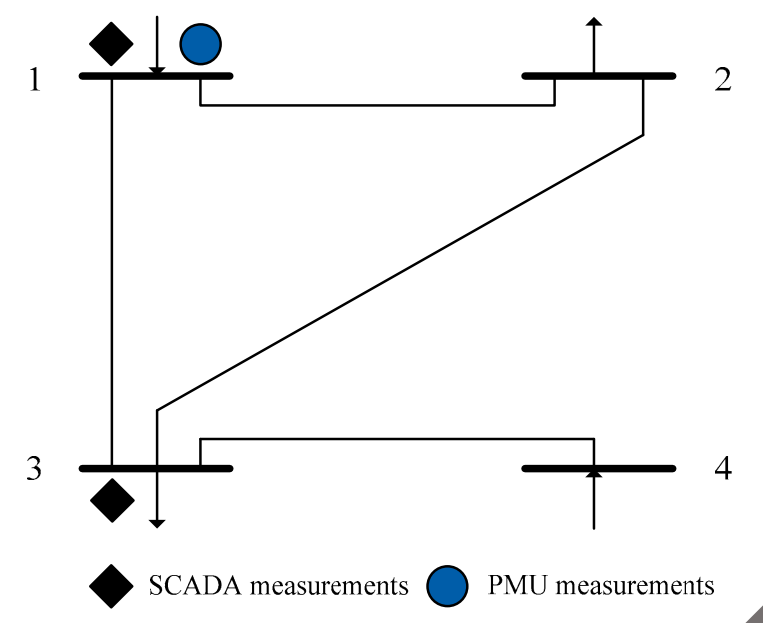

Figure 9. Four-node experiment test system.

Figure 10 is hardware design structure of the PMU used in the experiments. PMUs consist of signal conditioning modules, a synchronous clock module, data processing module and communication circuit. Signal conditioning modules consist of PT, CT and v detection circuits (PT uses SPT204A; CT uses SCT254AK). They convert analog signals of the power network into electrical signals which can be sampled and are suitable for ADC. Through DSP the data processing modules convert analog signals to digital signals. The synchronous clock module tracks synchronous 1PPS from the GPS, and generates trigger DSP synchronous sampling pulse signals. Power parameters such as phase angle, frequency, and voltage will be uploaded to the host computer via the communication circuit.

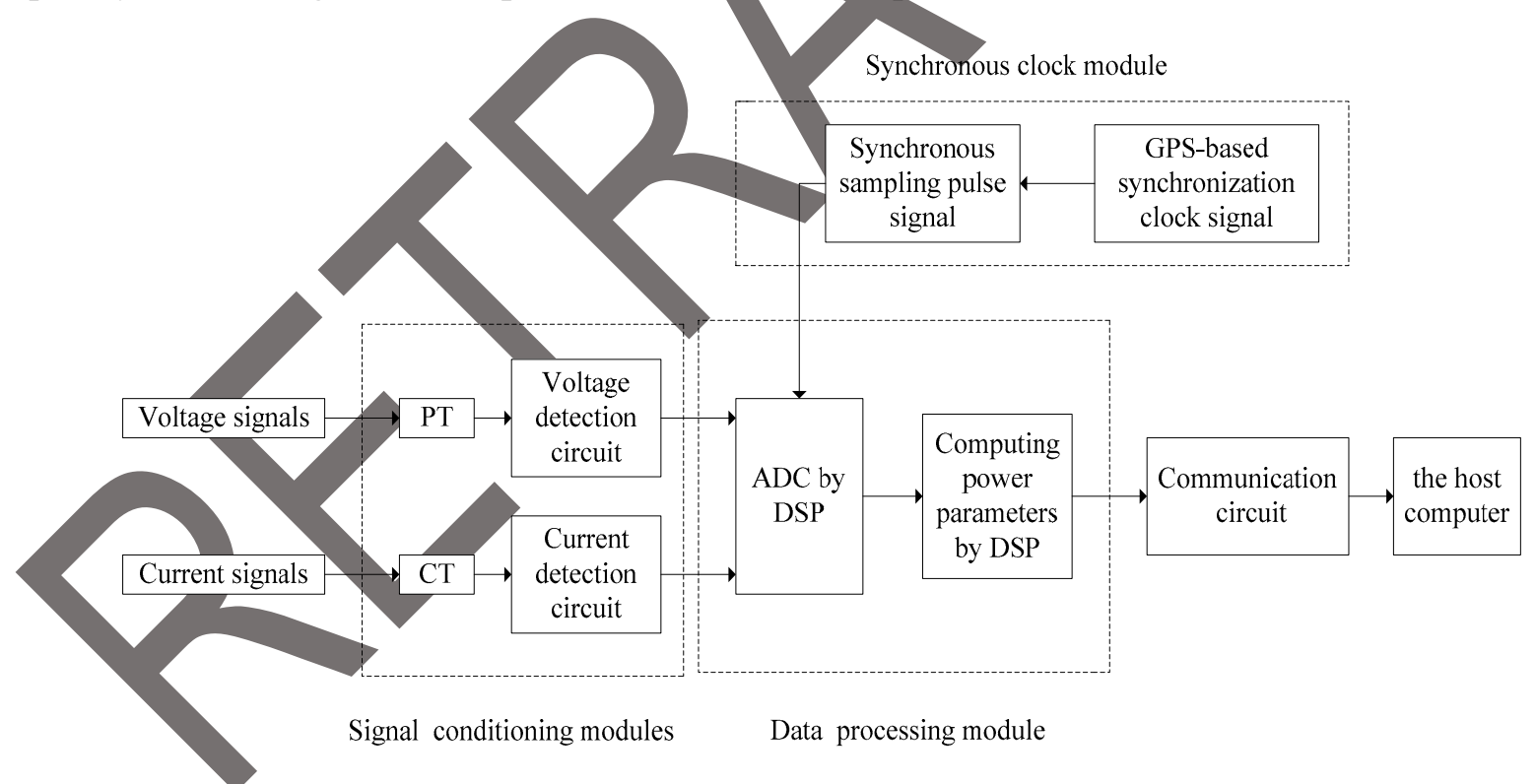

Figure 10. PMU design of a four-node experimental test system.

As shown in Figure 11, Different PMUs will use the same synchronized signal as sampling pulse trigger signal which is sent by the same control center, so the parameters' detection will have a very good time synchronization. In our experiments, the synchronized GPS clock module uses ATK-NEO-6M, which generates trigger DSP synchronous sampling pulse signal of executing ADC. It marks the sampled data processing results with a uniform high-accuracy time scale. 


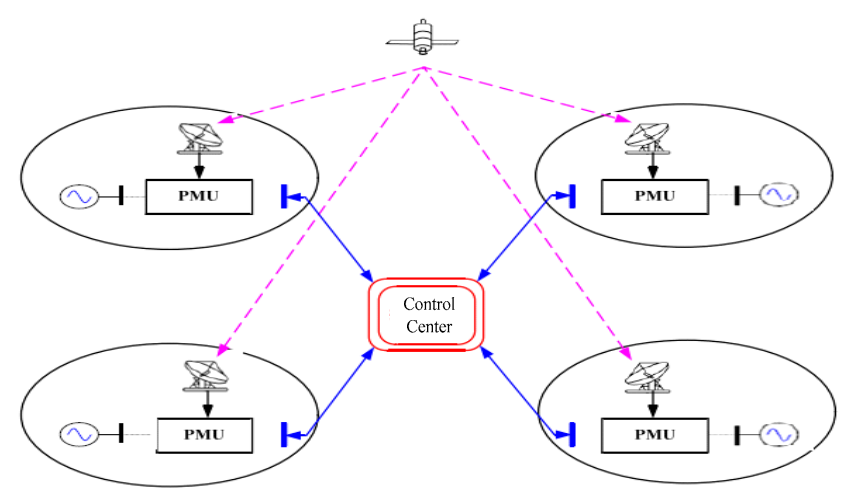

Figure 11. Synchronized PMU detection based on GPS signal.

STD of SCADA measurements is 0.01 and STD of PMU is given as 0.001 , and Figure 12 shows the estimation results of our four-node experiment test system. From the figure we can see the bus voltage estimation results and their errors. By repeating a lot of experiments, the proposed robust WLS method was proved to have very high accuracy and good computational efficiency; the accuracy of the bus voltage amplitude state estimation is $99.8 \%$ and the accuracy of the bus voltage angle estimation is about $99.4 \%$. The computational speed of the proposed method is much faster than the traditional WLS state estimation method.

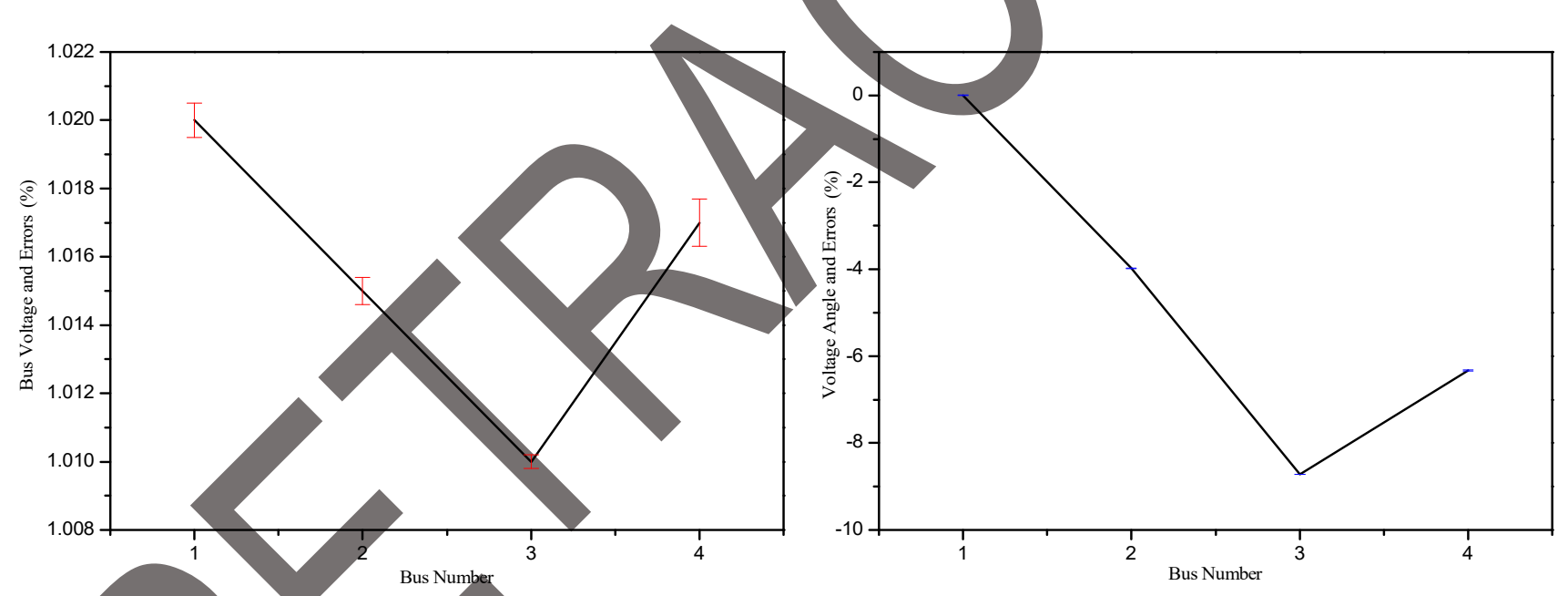

Figure 12. Estimation results of four-node experiment test system (a) Bus voltage estimation results and errors; (b) Bus voltage angle estimation results and errors.

In statistics, bias of an estimator is the difference between this estimator's expected value and the true value of the parameter being estimated. Suppose $\hat{x}$ is an estimator of parameter $x_{t}$. Then the bias of this estimator is defined as:

$$
\operatorname{Bias}[\hat{x}]=E[\hat{x}]-x_{t}=E\left[\hat{x}-x_{t}\right]
$$

When an estimator has zero bias it is called unbiased. Otherwise the estimator is said to be biased. Based on mathematical theory, from Equation (1) we can get:

$$
\operatorname{Bias}[\hat{x}]=\sum_{k=1}^{\infty}\left(\hat{x}-x_{t}\right) P_{k}
$$


where $P_{k}$ is the distribution probability. In statistics, there are problems for which it may be good to use an estimator with a small, but nonzero, bias since the property of mean-unbiasedness may be lost under nonlinear transformations. In this paper, we used a Monte Carlo simulation to research the statistical characteristics, and the estimation step number $N$ is 500 steps. The measurement inputs are randomly produced by:

$$
z=z_{0}(\mu+\sigma \times \operatorname{randn}(1,1))
$$

Figure 13 is the bias analysis of proposed the method using the Monte Cario method. It's clear from the figure that the bias is zero, so the estimator is unbiased. The other cases are also unbiased. Hence we can say the proposed robust WLS state estimators are unbiased.

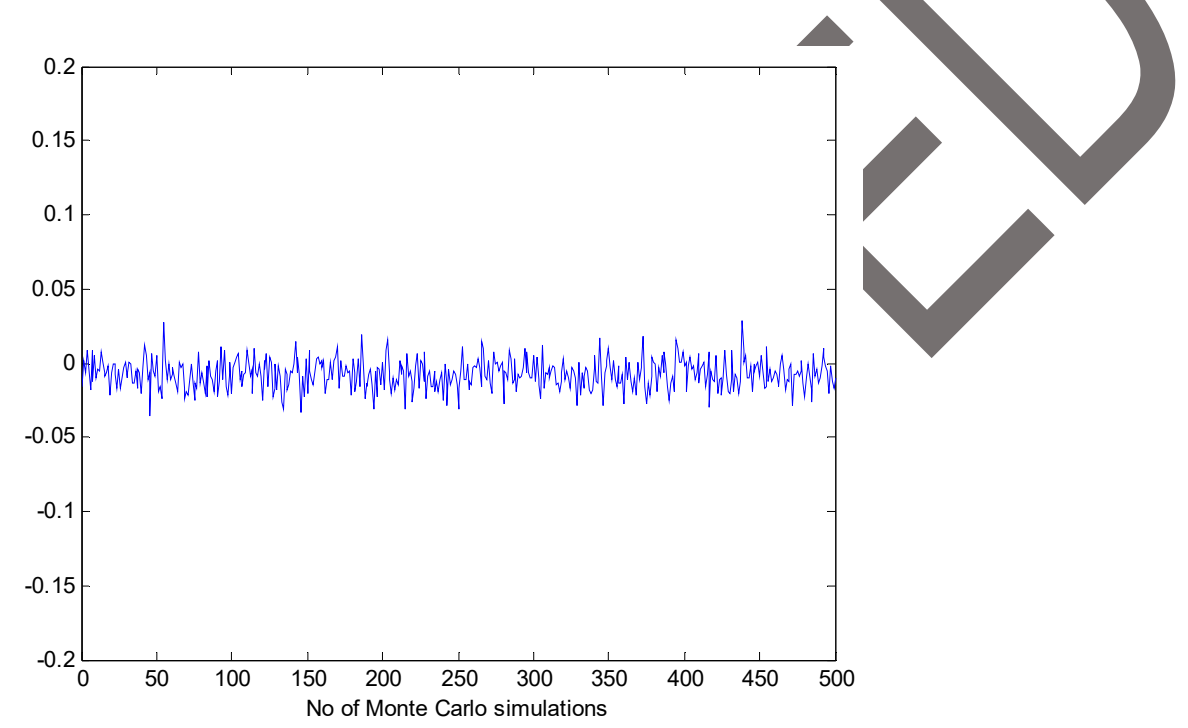

\section{Conclusions}

The method proposed in this paper analyzes in details the state estimation model by mixed measurements with WAMS and SCADA, and according to bad data and outliers in the measurement vector, researches a robust and high-efficiency WLS state estimation method. The proposed method takes into consideration measurement dependencies and updates the weighting factors automatically, therefore recognizing and eliminating bad data and outliers efficiently, and improves the robustness in the presence of bad measurements. This method's performance was tested on some IEEE bus systems and relative experiments. The results of simulations and experiments proved that the proposed method had good accuracy and high reliability and it can be used in state estimation of large scale and complicated power grids. The presented method can be extended to the analysis of other complex energy systems with multiple spatial-temporal scales like wind and solar energy as well.

\section{Acknowledgments}

This work was primarily supported by European Community 7th Framework Programme (Number: 909880), Chinese National Natural Science Foundation (Number: 50907011), Fujian Science Foundation for Distinguished Young Scholars (Number: 2012J06012). We also appreciate the 
support from Education office of Fujian province (Number: JA1108) and Fuzhou University (Number: 022222). The authors would like to thank all the reviewers for their advices and suggestions on improving this paper.

\section{Author Contributions}

Tao Jin coordinated the main theme of this paper and wrote the manuscript. Fuliang Chu developed the simulation models and performed experiments. Cong Ling discussed the research results and commented on the manuscript. Daniel Legrand Mon Nzongo partially completed the simulation. All the authors read and approved the final manuscript.

\section{Conflicts of Interest}

The authors declare no conflict of interest.

\section{References}

1. Phadke, A.G.; Moraes, R.M. The wide world of wide-area measurement. IEEE Power Energy Mag. 2008, 6, 52-65.

2. Yu, K.C.; Watson, N.R.; Arrillaga, J. Error analysis in static harmonic state estimation: A statistical approach. IEEE Trans. Power Deliv. 2005, 20, 1045-1050.

3. Jiang, W.Q.; Vittal, V.; Heydt, G.T. A distributed state estimator utilizing synchronized phasor measurements. IEEE Trans. Power Syst. 2007, 22, 563-571.

4. Wang, B.; He, G.Y.; Liu, K.C. A new scheme for guaranteed state estimation of power system. IEEE Trans. Power Syst. 2013, 28, 4875-4876.

5. Guo, Y.; Wu, W.C.; Zhang, B.M.; Sun, H.B. A fast solution for the lagrange multiplier-based electric power network parameter error identification model. Energies 2014, 7, 1288-1299.

6. Luque, J.; Gomez, I. The role of medium access control protocols in SCADA systems. IEEE Trans. Power Deliv. 1996, N, 1195-1200.

7. Thomas, M.S.; Kumar, P.; Chandna, V.K. Design, development, and commissioning of a supervisory control and data acquisition (SCADA) laboratory for research and training. IEEE Trans. Power Syst. 2004, 19, 1582-1588.

8. Ree, D.L.; Centeno, V.C.; Thorp, J.S.; Phadke, A.G. Synchronized phasor measurement applications in power systems. IEEE Trans. Smart Grid. 2010, 1, 20-27.

9. Zhong, Z.; Xu, C.; Billian, B.J.; Zhang, L.; Tsai, S.J.; Conners, R.W.; Centeno, V.C.; Phadke, A.G.; Liu, Y. Power system frequency monitoring network (FNET) implementations. IEEE Trans. Power Syst. 2005, 20, 1914-1921.

10. Yao, W.; Jiang, L.; Wu, Q.H.; Wen, J.Y.; Cheng, S.J. Delay-dependent stability analysis of the power system with a wide-area damping controller embedded. IEEE Trans. Power Syst. 2011, 26, 233-240.

11. Kamwa, I.; Samantaray, S.R.; Joos, G. Development of rule-based classifiers of rapid stability assessment of wide-area post-disturbances records. IEEE Trans. Power Syst. 2009, 24, 258-270. 
12. Song, H.L.; Wu, J.Y.; Wu, K. A wide-area measurement systems-based adaptive strategy for controlled islanding in bulk power systems. Energies 2014, 4, 2631-2657.

13. Dobakhshari, A.S.; Ranjbar, A.M. A circuit approach to fault diagnosis in power systems by wide area measurement system. Int. Trans. Elect. Energy Syst. 2013, 63, 272-1288.

14. Xu, W.; Wang, M.; Cai, J.; Tang, A. Sparse error correction from nonlinear measurements with applications in bad data detection for power networks. IEEE Trans. Sign. Proc. 2013, 61, 6175-6178.

15. Chen, J.; Abur, A. Placement of PMUs to enable bad data detection in state estimation. IEEE Trans. Power Syst. 2006, 21, 1608-1615.

16. Huang, Y.; Esmalifalak, M.; Nguyen, H.; Zheng, R.; Han, Z.; Li, H.; Song, L. Bad data injection in smart grid: attack and defense mechanisms. IEEE Comm. Mag. 2013, 51, 27-33.

17. Brameller, A.; Karaki, S.H. Power-system state estimation using linear programming. Proc. Inst. Electr. Eng. 1979, 126, 246-247.

18. Haughton, D.A.; Heydt, G.T. A linear state estimation formulation for smart distribution systems. IEEE Trans. Power Syst. 2013, 28, 1187-1195.

19. Yang, T.; Sun, H.; Bose, A. Transition to a two-level linear state estimator-part I: Architecture. IEEE Trans. Power Syst. 2011, 26, 46-53.

20. Yang, T.; Sun, H.; Bose, A. Transition to a two-level linear state estimator-part II: Algorithm. IEEE Trans. Power Syst. 2011, 26, 54-62.

21. Huang, C.H.; Lee, C.H.; Shih, K.R.; Wang, Y.J. Bad data analysis in power system measurement estimation using complex artificial neural network based on the extended complex Kalman filter. European Trans. Electr. Power. 2010, 20, 1082-1100.

22. Singh, D.; Pandey, J.P.; Chauhan, D.S. Topology identification, bad data processing, and state estimation using fuzzy pattern matching. IEEE Trans. Power Syst. 2005, 20, 1570-1579.

23. Zhang, J.H.; Welch, G.; Bishop, G.; Huang, Z.Y. A two-stage kalman filter approach for robust and real-time power system state estimation. IEEE Trans. Sust. Energy 2014, 2, 629-636.

24. Yu, K.C.; Watson, N.R. An approximate method for transient state estimation. IEEE Trans. Power Deliv. 2007, 22, 1680-1687.

25. Kundu, P.; Pradhan, A.K. Wide area measurement based protection support during power swing. Int. J. Electr. Power Energy Syst. 2014, 635, 546-554.

(C) 2015 by the authors; Micensee MDPI, Basel, Switzerland. This article is an open access article distributed under the terms and conditions of the Creative Commons Attribution license (http://creativecommons.org/licenses/by/4.0/). 